\title{
Dissolution enhancement of glimepiride dispersion using glyceryl monostearate and $\beta$-cyclodextrin as carrier
}

\author{
Md. Abu Shuaib Rafshanjani1 ${ }^{1}$ *Md. Mofizur Rahman'1, Shumaia Parvin², and Md. Abdul Kader ${ }^{2}$ \\ ${ }^{1}$ Department of Pharmacy, Bangladesh University, 15/1, Iqbal Road, Mohammadpur, Dhaka-1207, Bangladesh \\ ${ }^{2}$ Department of Pharmacy, University of Rajshahi, Rajshahi 6205, Bangladesh
}

\begin{abstract}
Glimepiride is an antidiabetic drug of sulfonylurea group and indicated for the treatment of type 2 diabetes mellitus. The present study was conducted to enhance the dissolution rate of glimepiride solid lipid nano particle dispersions using hot homogenization method and glimepiride solid dispersion by precipitation method. Solid lipid nanoparticles have been used as suitable carriers for delivery of drug with poor solubility. In this investigation glyceryl monostearate and stearic acid were used as solid lipid, Lutrol F68 as surfactant, Tween 80 as stabilizer and the used polymer were urea crystal and $\beta$-cyclodextrin. Three formulations were prepared in different ratios for two methods and were designated as GMLN1 to GMLN3 in case of hot homogenization method and GMP1 to GMP3 for precipitation method. The evaluation of all the dispersions were done by in vitro dissolution studies using US Pharmacopeia type II apparatus (paddle method) in $900 \mathrm{ml}$ distilled water at $50 \mathrm{rpm}$ to a temperature of $37^{\circ} \mathrm{C} \pm 0.5^{\circ} \mathrm{C}$ for 45 minutes. In situ and externally sink method revealed the release pattern of drug was found to follow zero order, first order and KorsmeyerPeppas equations. Improved dissolution profile was observed in all the solid lipid nano particle dispersions as compared to pure drug as well as market preparation. Thus, glyceryl monostearate and $\beta$-cyclodextrin can be successfully used as carrier for improvement of dissolution and bioavailability of glimepiride.
\end{abstract}

Key Words: Glimepiride, solid lipid nano particles, dissolution rate, hot homogenization method, precipitation method.

\section{INTRODUCTION}

The aqueous solubility of a drug is a key determinant of its oral bioavailability. There are some drugs for which solubility has presented a challenge to the development of a suitable formulation for oral administration. Drug absorption, sufficient and reproducible bioavailability, pharmacokinetic profile of orally administered drug substances is highly dependent on aqueous solubility of that compound (Jatwani et al., 2012; Wagh and Wagh, 2015). More than $40 \%$ of new drugs have poor aqueous solubility, resulting in unsatisfactory oral drug delivery. Thus, increased solubility, dissolution rate and bioavailability of drug have a very crucial role in the process of drug development (Yogesh et al., 2011). Besides this, BCS II drugs are very much prone to dissolution-rate limited gastrointestinal absorption (Shohin et al., 2011; Reddy et al., 2011). Hence, by improving drug dissolution in aqueous medium, various formulation techniques can accelerate a parallel improvement in bioavailability (Wei and Lobenber, 2006; Kawabata et al., 2011; Kawakami, 2012).

Drug dissolution enhancement has been extensively studied over the past decade through different techniques (Ahuja et al., 2007; Blagden et al., 2007; Cilurzo et al., 2008; Makar et al., 2013). Among these, the SD technique is the most successful in improving the dissolution and bioavailability of poorly soluble active pharmaceutical ingredients because it is very simple and economic (Leuner and Dressman, 2000; Chiou and Rigelman, 1971; Serajuddin, 1999). Solid lipid nanoparticles (SLN) are aqueous colloidal dispersions, is made up of solid biodegradable lipids having a lot of the advantages with a limited drawbacks

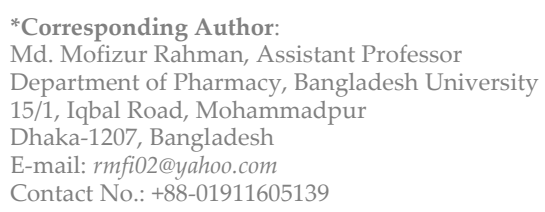

(Swathi et al., 2010; Sarathchandiran, 2012).

Glimepiride (GMP) is 3-ethyl-4-methyl-N-\{2-[4(\{[(4methylcyclohexyl) carbamoyl] amino\} sulfonyl) phenyl] ethyl, is an antidiabetic drug originated from the second generation sulphonyl urea group and stimulates the release of insulin. It is also classified as class II drug according to biopharmaceutical classification system and used for treatment of non-insulin-dependent diabetes mellitus (Frick et al., 1998; Massimo, 2003). In acidic and neutral aqueous media, glimepiride shows very poor solubility at $37^{\circ} \mathrm{C}(<0.004 \mathrm{mg} / \mathrm{ml})$. Solubility of drug is slightly increased by $0.02 \mathrm{mg} / \mathrm{ml}$ in media having $\mathrm{pH}>7$. Poor dissolution and unsatisfied bioavailability of this drug are the results of this poor aqueous solubility (Frick et al., 1998). Previous reports on this drug revealed that dissolution rate is increased through formation of inclusion complex with cyclodextrin (Ammar et al., 2006) or preparation of solid dispersions using either water soluble carriers (Boregowda et al., 2011; Rajpurohit et al., 2011), insoluble carriers (Kiran et al., 2009; Reven et al., 2010; Vidyadhara et al., 2011) or preparation of spray congealed microparticles (Ilić et al.,, 2009). However, for improving its bioavailability only a few attempts have been made. Hence, the present investigation was done to improve the dissolution rate as well as bioavaibility of glimepiride by the preparation of solid lipid nano particle dispersion using glyceryl monostearate and stearic acid as solid lipid and by formation of inclusion complex with $\beta$ cyclodextrin and urea crystal.

\section{MATERIALS AND METHODS}

\section{Materials}

Glimepiride was a gift sample from Beximco Pharmaceuticals, Dhaka, Bangladesh. Glyceryl monostearate and stearic acid were procured from Balami Fine Chemicals, India. Tween 80 and lutrol F-68 (poloxamer) were received from Merk chemicals, Mumbai, India. Betacy- 
clodextrin, urea crystals were obtained from BASF, Germany. All other reagents and solvent used were of analytical grade.

\section{Preparation of solid lipid nanoparticle dispersions by hot homogenization method \\ Solid lipid nanoparticle dispersions were prepared by hot homogenization technique (Mehnert and Mader, 2001). Lipid was melted at temperature ten degrees above its melting point then glimepiride was added to the melted lipid. The dispersion was kept at the same temperature until it is appeared as optically clear. Tween 80 as stabilizer was dissolved in distilled water and heated at the same temperature of lipid mixture. Hot surfactant solution was then added to the melted lipid-drug mixture and emulsified by a homogenizer at $12000 \mathrm{rpm}$ for 2 hours. The formulation was then removed from water bath and the dispersion of SLN was mixed gently at room temperature (table 1).}

\section{Preparation of solid dispersions by precipitation method} For the preparation of solid dispersion glimepiride (GMP) was dissolved in ethanol and $\beta$-cyclodextrin $(\beta-C D)$ was dissolved in distilled water at room temperature (Sapkal et al., 2007). Different ratios of drug, $\beta-C D$ and urea crystal were taken (table 2). The mixture was stirred at room temperature for one hour and then slowly evaporated on a boiling water bath. The precipitates as a crystalline powder was pulverized followed by screening through sieve No. 100 and stored in a desiccator till free from any traces of the organic solvent.

\section{In vitro dissolution studies}

In vitro dissolution studies of the pure drug, solid dispersions and market preparations were carried out to estimate the cumulative percentage of drug release with respect to time (Khan and Rhodes, 1975). Distilled water $(900 \mathrm{ml})$ was placed in each vessel of the USP type II rotating paddle dissolution apparatus (Veego Vda 6DR, Germany). Two capsules or SDs granules (equivalent to $2 \mathrm{mg}$ of drug) were placed in each vessel and the medium was allowed to equilibrate at $50 \mathrm{rpm}$ to a temperature of $37 \pm 0.5^{\circ} \mathrm{C}$ for 45 minutes. $10 \mathrm{ml}$ of the sample was withdrawn at definite time interval $(5,10,20,30$, and 45 minutes) consecutively and replaced by fresh media to maintain the sink condition. The absorbances of sample were measured at $\lambda_{\max } 236 \mathrm{~nm}$ on a UV-Visible spectrophotometer (UVmini-1240, Shimadzu, Kyoto, Japan).

\section{Release kinetic modeling}

The release pattern of drug from the dosage forms has been described by a number of kinetic models. As we know that the drug release, in vivo performance may be altered by qualitative and quantitative changes in a formulation thus in vitro drug dissolution data can be used in the rational development of controlled release formulations (Dash et al., 2010).

\section{Zero-order model}

In zero order kinetics the drug release rate is independent of the initial concentration (Hadjiioannou et al., 1993) i.e., whatever the amount of drug at absorption site release rate remains the same.

\section{$\mathbf{C t}=\mathrm{C}_{0}+\mathrm{K}_{0} \mathbf{t}$}

Where, $\mathrm{Ct}$ is the amount of drug dissolved in time $\mathrm{t}, \mathrm{C}_{0}$ is the initial amount of drug in the solution (most times, $\mathrm{C}_{0}=$ $0), K_{0}$ is the zero-order release rate constant expressed in concentration / time and $t$ is the time in hours. The data obtained from in vitro drug release studies were plotted as cumulative amount of drug released versus time.

\section{First-order model}

The rate of appearance of drug at the absorption site can be measured by an exponential process (Bourne, 2002) in which the rate of release is only dependent on the remaining drug concentration.

$$
\log C=\log C_{0}-\frac{k t}{2.303}
$$

Where, $\mathrm{C}$ is the concentration of drug at time $\mathrm{t}, \mathrm{C}_{0}$ is the initial concentration, $\mathrm{K}$ is the first order release rate constant expressed in time- ${ }^{1}$. The data are plotted as log cumulative percentage of drug remaining versus time which would obtain a straight line with a slope of $\mathrm{K} / 2.303$

\section{Korsmeyer-Peppas Model}

To describe the drug release phenomenon from polymeric systems, the Korsmeyer- Peppas equation (Korsmeyer et al., 1983) is used usually.

$$
\log \left(\frac{M_{t}}{M_{f}}\right)=\log k+n \log t
$$

Where $\mathrm{M}_{\mathrm{t}}$ is the amount of drug release at time $t ; \mathrm{M}_{\mathrm{f}}$ is the amount of drug release after infinite time; $\mathrm{k}$ is a release rate constant; $\mathrm{n}$ is the diffusional exponent indicating the mechanism of drug release. The log of percentage drug dissolved is plotted against log time for each formulation. A value of $\mathrm{n}=0.45$ indicates Fickian diffusion (case-I) release; $0.45<\mathrm{n}<0.89$ for non-Fickian diffusion (anomalous) release; $\mathrm{n}=0.89$ case-II transport and $\mathrm{n}>0.89$ indicates super case II release.

\section{Mean Dissolution Time (MDT)}

Mean Dissolution Time (MDT) value is a model independed approach and can be calculated from dissolution data using Mockel and Lippold equation (Mockel and Lippold, 1993). This value can be used to characterize the drug release rate from the dosage form and the retarding efficiency of the polymer.

$$
\mathbf{M D T}=\left(\frac{\mathbf{n}}{\mathbf{n}+\mathbf{1}}\right) \mathbf{k}^{-1 / \mathbf{n}}
$$

Where, $\mathrm{n}$ is release exponent and $\mathrm{k}$ is release rate constant.

\section{Successive fractional dissolution time}

To characterize the drug release rate in different experimental condition, $\mathrm{T}_{25 \%}$ (time required for $25 \%$ drug release), $\mathrm{T}_{50 \%}, \mathrm{~T}_{80} \%$ and $\mathrm{MDT}$ were calculated from dissolution data according to the equations: $\mathrm{T}_{25} \%=$ $(0.25 / \mathrm{k})^{1 / \mathrm{n}}, \mathrm{T}_{50 \%}=(0.5 / \mathrm{k})^{1 / \mathrm{n}}, \mathrm{T}_{80} \%=(0.8 / \mathrm{k})^{1 / \mathrm{n}}$ and MDT $=$ $(n / n+1) k^{-1 / n}$ where $n$ is a release exponent and $k$ is the release rate constant for Korsmeyer- Peppas equation.

\section{Statistical analysis}

A one way of analysis of variance (ANOVA) was used to analyze the dissolution data obtained for each batch of formulation to compare the drug release rate comparison of successive dissolution time $\left(\mathrm{T}_{25} \%, \mathrm{~T}_{50} \%, \mathrm{~T}_{80} \%\right.$, MDT). A confidence limit of $\mathrm{P}<0.05$ was fixed and theoretical calculated values of $\mathrm{F}$ (Fcrit and Fcal) were compared for the interpretation of results. 


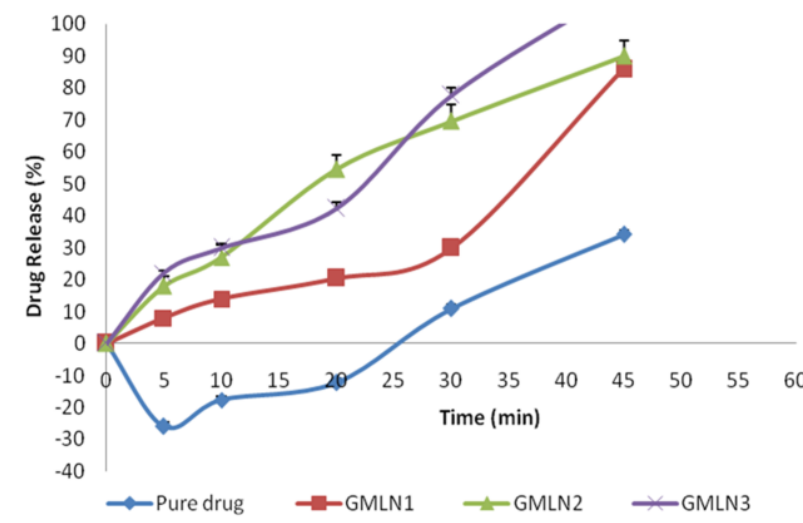

(a) Glyceryl monosterate and stearic acid containing formulations

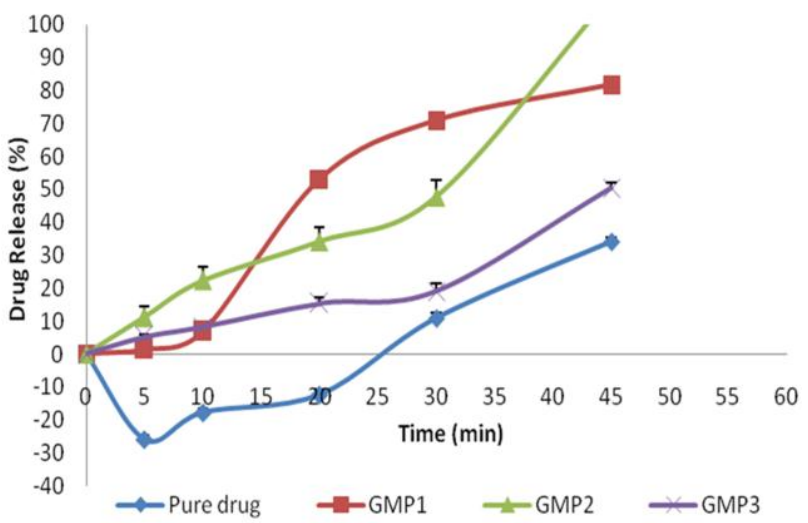

(b) $\beta$-cyclodextrin and urea crystal containing formulations

Figure 1: Zero orders plots of glimepiride SLNP formulations with different ratio of lipid and polymer in distilled water.

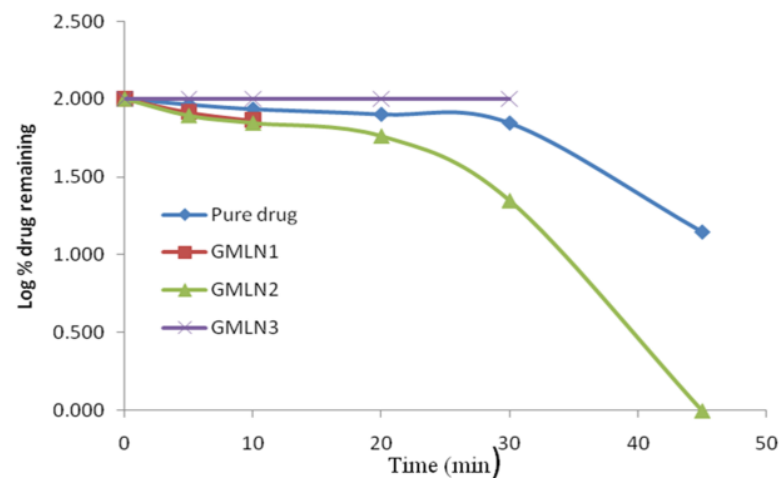

(a) Glyceryl monosterate and stearic acid containing formulations

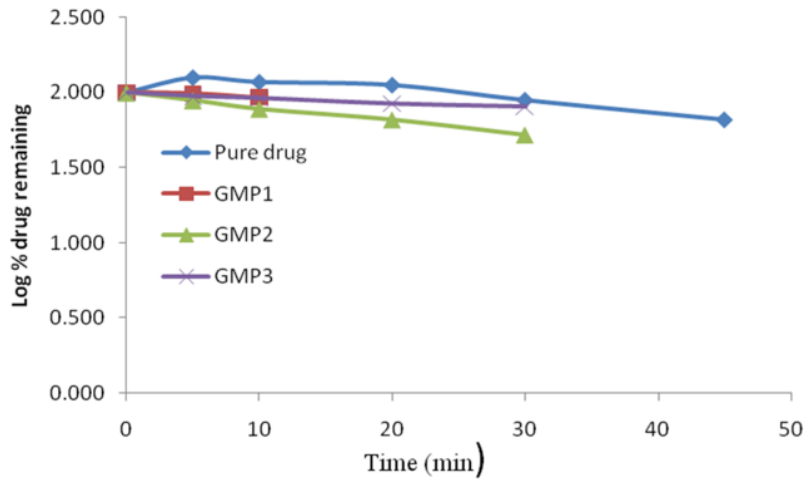

(b) $\beta$-cyclodextrin and urea crystal containing formulations

Figure 2: First orders plots of glimepiride SLNP formulations with different ratio of lipid and polymer in distilled water.

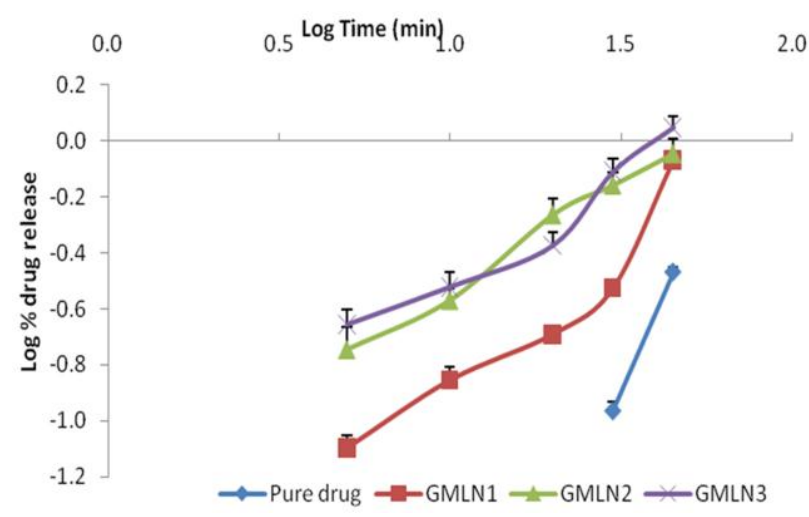

(a) Glyceryl monosterate and stearic acid containing formulations

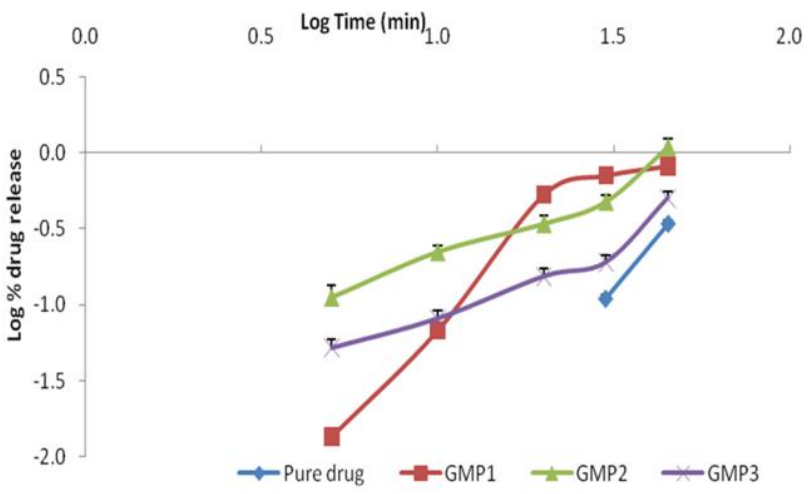

(b) $\beta$-cyclodextrin and urea crystal containing formulations

Figure 3: Korsmeyer kinetic plots of glimepiride SLNP formulations with different ratio of lipid and polymer in distilled water. 
Table 1: Formulation of glimepiride solid lipid nanoparticle dispersions through hot homogenization method.

\begin{tabular}{ccccc}
\hline Formulation Code & Solid lipid (200mg) & Surfactant, Lutrol F-68 (mg) & Stabilizer, Tween $\mathbf{8 0}(\mathbf{m l})$ & Water $(\mathbf{m l})$ \\
\hline GMLN1 & Stearic acid & 400 & 1 & 15 \\
GMLN2 & Stearic acid & 400 & 1.5 & 15 \\
GMLN3 & Glyceryl monostearate & 400 & 1 & 15 \\
\hline
\end{tabular}

Table 2: Formulation of glimepiride solid dispersions through precipitation method.

\begin{tabular}{ccc}
\hline Formulation Code & Drug-Polymer composition & Drug-polymer ratio \\
\hline GMP1 & GMP- $\beta C D$ & $1: 1$ \\
GMP2 & GMP- $\beta$ CD & $1: 3$ \\
GMP3 & GMP-urea crystal & $1: 1$ \\
\hline
\end{tabular}

Table 3: Regression coefficient $\left(\mathrm{r}^{2}\right)$ of different kinetic model and diffusion exponent $(\mathrm{n})$ of Korsmeyer-Peppas model of glimepiride release from different SLNP formulations.

\begin{tabular}{ccccc}
\hline Formulations & Zero Order $\left(\mathbf{r}^{\mathbf{2}}\right)$ & First Order $\left(\mathbf{r}^{2}\right)$ & Korsmeyer $\left(\mathbf{r}^{2}\right)$ & Korsmeyer (n) \\
\hline GMLN1 & 0.877 & 0.789 & 0.901 & 0.965 \\
GMLN2 & 0.972 & 0.837 & 0.990 & 0.762 \\
GMLN3 & 0.826 & 0.909 & 0.942 & 0.741 \\
GMP1 & 0.915 & 0.880 & 0.938 & 0.986 \\
GMP2 & 0.936 & 0.994 & 0.954 & 0.946 \\
GMP3 & 0.907 & 0.761 & 0.931 & 0.955 \\
\hline
\end{tabular}

Table 4: Percent release of glimepiride from SLNP formulations with different carrier.

\begin{tabular}{|c|c|c|c|c|c|c|c|}
\hline \multirow{2}{*}{ Time (min) } & \multicolumn{7}{|c|}{ Percent of drug released } \\
\hline & Pure Drug & GMLN1 & GMLN2 & GMLN3 & GMP1 & GMP2 & GMP3 \\
\hline 0 & 0 & 0 & 0 & 0 & 0 & 0 & 0 \\
\hline 5 & -25.90 & 8.00 & 18.00 & 22.00 & 1.36 & 11.20 & 5.21 \\
\hline 10 & -17.73 & 14.00 & 27.00 & 30.00 & 6.82 & 22.31 & 8.13 \\
\hline 20 & -12.27 & 20.45 & 54.55 & 42.27 & 53.18 & 34.09 & 15.40 \\
\hline 30 & 10.90 & 30.00 & 69.55 & 77.73 & 70.91 & 47.73 & 19.09 \\
\hline 45 & 34.09 & 85.90 & 90.00 & 111.82 & 81.82 & 109.09 & 50.45 \\
\hline
\end{tabular}

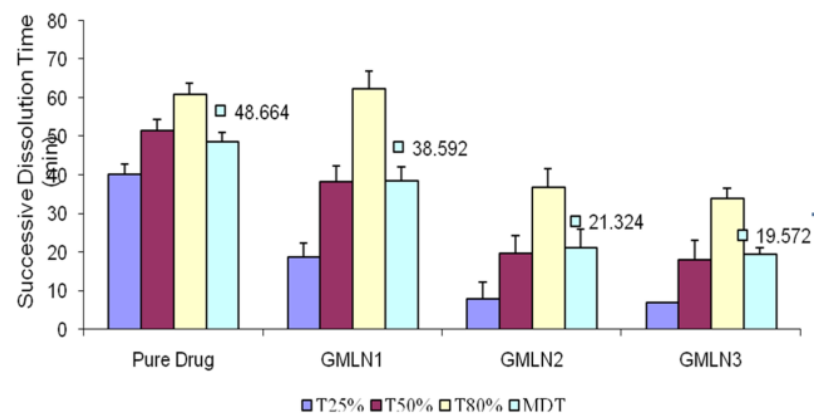

(a) Glyceryl monosterate and stearic acid containing formulations

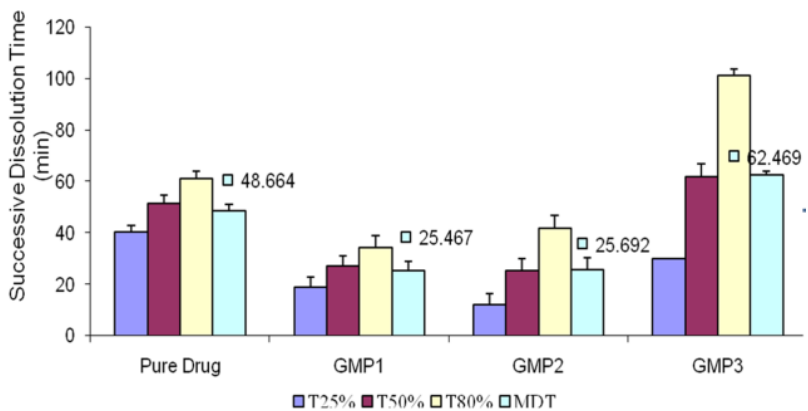

(b) $\beta$-cyclodextrin and urea crystal containing formulations

Figure 4: Successive fractional dissolution time and MDT values of glimepiride SLNP formulations with different ratio of lipid and polymer in distilled water.

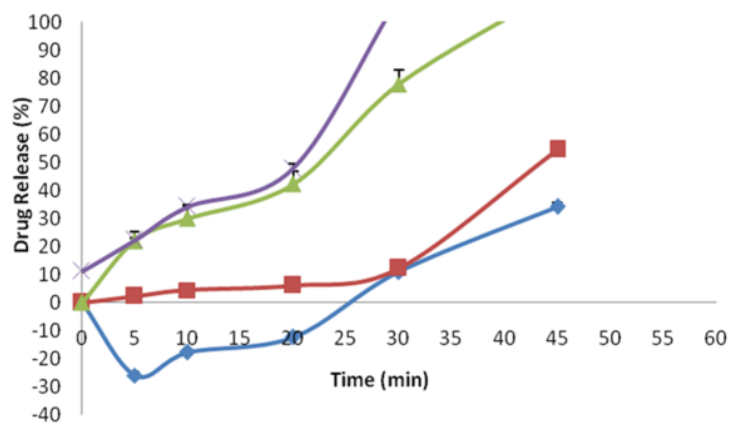

Figure 5: Optimization of release profile of glimepiride (pure drug, market preparation, GMLN3 and GMP2). 


\section{RESULTS}

Different combination of lipid and polymer were used to provide glimepiride solid lipid nano particle dispersions. The dissolution profile of all the formulations were fitted to zero order, first order and Korsmeyer-Peppas model to ascertain the kinetic modeling of drug release (figure 1, 2 and 3 , respectively). Table 3 showed data analysis of release profiles according to different kinetic models. It was found that the in vitro drug release was best explained by Korsmeyer-Peppas equation as the plots showed good linearity $\left(\mathrm{r}^{2}=0.901\right)$ followed by zero order $\left(\mathrm{r}^{2}=0.972\right)$ and first order $\left(\mathrm{r}^{2}=0.909\right)$. The values of $\mathrm{n}$ (release exponent) for all formulations studied here were between 0.741 to 0.986 indicating an anomalous behavior corresponding to diffusion, erosion and swelling mechanisms of drug release. Solid dispersions formulated with different carriers exhibited significant improvement in the dissolution (table 4) of glimepiride as compared to pure drug. GMLN3 and GMP2 showed highest dissolution rate that is $111.82 \%$ and $109.09 \%$ respectively whereas the release percentage of pure drug was $34.09 \%$. Successful fractional dissolution time and MDT values calculated for all the SLNPs were illustrated in figure 4 . This finding was also corroborated with the drug release process indicating that GMLN3 and GMP2 had least MDT values $19.572 \mathrm{~min}$ and $25.692 \mathrm{~min}$ respectively compared to other formulations. In order to evaluate the drug dissolution profile with optimized formulation and market preparation of glimepiride as well as pure drug, it was interesting to compare the results of the dissolution tests (figure 5). The results revealed that optimized formulation (GMLN3, GMP2) showed highest drug release rate than market preparation.

\section{DISCUSSION}

The kinetic modeling of drug release shows that these models have been established to describe the relationship between drug dissolution and drug release patterns mathematically. The corresponding plot (log cumulative percent drug release versus time) for the Korsmeyer Peppas equations indicated a good linearity $\left(\mathrm{r}^{2}=0.901\right)$. Value of the release exponent ' $n$ ' was in between 0.741 and 0.986, indicating anomalous diffusion, i.e., drug release was controlled by more than one process. Reddy et al (2003) observed similar results with a matrix tablet of nicorandil with an $\mathrm{n}$ value of 0.71 . From the proposed formulation, it is evident that when excipient glyceryl monostearate is used in hot homogenization method, the drug release increases significantly from solid lipid nano particles due to wetting ability and converting crystalline to amorphous form which enhance the solubility. This lipid also increases the entrapment efficiency of glimepiride by increasing the viscosity of medium which prevents rapid diffusion of glimepiride into bulk of medium (Yang, 2000). When this polymer is used the release percentage has been increased from $34.09 \%$ (pure drug) to $111.82 \%$. This results are clear indication that glyceryl monostearate has lower drug retarding ability which is confirmed by smaller MDT value (19.572 min). Lutrol F-68 and glyceryl monostearate or stearic acid can act as gelling agent at high concentration and it affect the drug dissolution from solid lipid nano particles. When stearic acid is used instead of glyceryl monostearate drug release was almost near $(90.00 \%)$ to that of glyceryl monostearate.

In case of precipitation method, when $\beta$-cyclodextrin and glimepiride is formulated as 1:1 ratio, the drug release rate is $81.82 \%$, but in case of $1: 3$ ratio, the release rate is increased to $109.09 \%$. On the other hand, when urea crystal is used instead of $\beta$-cyclodextrin, then the drug release rate is $50.45 \%$ which is not as high as betacyclodextrin containing formulation. So, it is clear that the polymer $\beta$-cyclodextrin in higher amount used in precipitation method can give very much better dissolution rate. It was verified that the presence of $\beta$ cyclodextrin into polymeric drug delivery systems can also influence the drug release mechanism by Bibby et al (Bibby et al., 2000). In the present study, the results evidenced that release profiles of these formulations were only slightly slower than those containing glyceryl monosterate. This is probably due to an inclusion process of the glimepiride molecule in $\beta$-cyclodextrin, which may be considered energetically favoured when compared to inclusion of drugs, namely ibuprofen (Bragga et al., 2003). So, the present study confirms that solid lipid nanoparticles of glimepiride with lipid, surfactant and stabilizer and precipitated formulation from large amount of $\beta$ cyclodextrin can be formulated as tablet with better dissolution characteristics.

\section{CONCLUSION}

The results of the present study provide useful information on the type of lipid and polymer that should be employed on the formulation of glimepiride solid lipid nano particle dispersion. The release mechanism of glimepiride from each formulation tested was evaluated in the light of zero order, first order and KorsmeyerPeppas kinetic models. The Korsmeyer-Peppas model fits with the in vitro dissolution data and gives an insight into the possible drug release mechanism by Non-Fickian diffusion. In-vitro drug release through the USP type- $\Pi$ apparatus from the prepared SLNPs containing glyceryl monostearate is much higher $(111.82 \%)$ than the pure drug $(34.09 \%)$ by hot homogenization method. Again in case of precipitation method the formulation containing $\beta$-cyclodextrin provide higher drug release $(109.09 \%)$ Hence glimepiride SLNPs formulated with glyceryl monostearate and $\beta$-cyclodextrin enhance the dissolution rate as well as it will enhance the bioavailability of the drug and will increase the patient compliance.

\section{ACKNOWLEDGEMENT}

The authors are thankful to Beximco Pharmaceuticals Ltd, Dhaka, Bangladesh for providing the gift sample of glimepiride and to the Chairman, Department of Pharmacy, Bangladesh University, Dhaka, Bangladesh for providing necessary support to carry out this research work. This experiment was also supported by the Department of Pharmacy, University of Rajshahi, Rajshahi 6205, Bangladesh.

\section{REFERENCES}

Ahuja, N., Katare, OP. and Singh, B. (2007). Studies on dissolution enhancement and mathematical modeling of drug release of a poorly water-soluble drug using water-soluble carriers. European Journal

Pharmaceutics and Biopharmaceutics, Volume 65, Issue 1, Pages 26-38. [DOI]

Ammar, HO., Salama, HA., Ghorab, M. and Mahmoud, AA. (2006). Formulation and biological evaluation of glimepiride-cyclodextrinpolymer systems. International Journal of Pharmaceutics, Volume 309, Issue 1-2, Pages 129-138. [DOI]

Bibby, DC., Davies, NM. and Tucker, IG. (2000). Mechanisms by which cyclodextrins modify drug release from polymeric drug delivery systems. International Journal of Pharmaceutics, Volume 197, Issue 1-2, Pages 1-11. [DOI]

Blagden, N., Matas, DM., Gavan, PT. and York, P. (2007). Crystal engineering of active pharmaceutical ingredients to improve solubility 
and dissolution rates. Advanced Drug Delivery Reviews, Volume 59, Issue 7, Pages 617-630. [DOI]

Boregowda, SS., Rao, BPR., Jayarama, RA. and Guruchar, NLV. (2011) Application of water- soluble/ dispersible polymeric carriers in drug dissolution modulation. Asian Journal of Pharmaceutical Sciences, Volume 6, Issue 1, Pages 26-35.

Bourne, DW. (2002). Pharmacokinetics. In GS. Banker, CT. Rhodes, Modern Pharmaceutics (4th ed., pp. 67-92). New York, Marcel Dekker Inc. [DOI]

Braga, SS., Goncalves, IS., Herdtweck, E. and Teixeira-Dias, JJC. (2003) Solid state inclusion compound of S-ibuprofen in b-cyclodextrin: structure and characterization. New Journal of Chemistry, Volume 3, Pages 597-601. [DOI]

Chiou, WL. and Rigelman, S. (1971). Pharmaceutical application of solid dispersion system. Journal of Pharmaceutical Sciences, Volume 60 Issue 9, Pages 1281-1302. [DOI]

Cilurzo, F., Selmin, F., Minghetti, P., Gennari, CGM., Demartin, F. and Montanari, L. (2008). Characterization and physical stability of fastdissolving microparticles containing nifedipine. European Journal of Pharmaceutics and Biopharmaceutics, Volume 68, Issue 3, Pages 579588. [DOI]

Dash, S., Murthy, PN., Nath, L. and Chowdhuri, P. (2010). Kinetic modeling on drug release from controlled drug delivery systems. Acta Poloniae Pharmaceutica-Drug Research, Volume 67, Issue 3, Pages $217-$ 223.

Frick, A., Moller, H. and Wirbitzki, E. (1998). Biopharmaceutical characterization of oral immediate release drug products. In vivo / in vitro comparison of phenoxy methyl penicillin potassium, glimepiride and levofloxacin. European Journal of Pharmaceutics and Biopharmaceutics, Volume 46, Issue 3, Pages 305-311. [DOI]

Hadjiioannou, TP., Christian, GD. and Koupparis, MA. (1993). Quantitative Calculations in Pharmaceutical Practice and Research (pp. 345 348). New York, VCH Publishers Inc.

Ilić, I., Dreu, R., Burjak, M., Homar, M., Kerč, J. and Srčič, S. S. (2009). Microparticle size control and glimepiride microencapsulation using spray congealing technology. International Journal of Pharmaceutics, Volume 381, Issue 2, Pages 176-183. [DOI]

Jatwani, S., Ran, CA., Singh, G. and Aggarwal, G. (2012). An overview on solubility enhancement techniques for poorly soluble drugs and solid dispersion as an eminent strategic approach. International Journal of Pharmaceutical Science and Research, Volume 3, Issue 4, Pages 942-956.

Kawakami, K. (2012). Modification of physicochemical characteristics of active pharmaceutical ingredients and application of supersaturatable dosage forms for improving bioavailability of poorly absorbed drugs. Advanced Drug Delivery Reviews, Volume 64, Issue 6, Pages 480- 495 [DOI]

Kawabata, Y., Wada, K., Nakatani, M., Yamada, S. and Onoue, S. (2011). Formulation design for poorly water-soluble drugs based on biopharmaceutics classification system: basic approaches and practical applications. International Journal of Pharmaceutics, Volume 420, Issue 1, Pages 1-10. [DOI]

Khan, CA. and Rhodes, CT. (1975). The concept of dissolution efficiency. Journal of Pharmacy and Pharmacology, Volume 27, Issue 1, Pages 4849. [DOI]

Kiran, T., Shastri, N., Ramakrishna, S. and Sadanandam, M. (2009). Surface solid dispersion of glimepiride for enhancement of dissolution rate. International Journal of PharmTech Research, Volume 1, Issue 3, Pages 822-831.

Korsmeyer, RW., Gurry, R., Doelker, E., Buri, P. and Peppas, NA. (1983) Mechanisms of solute release from porous hydropjilic polymers. International Journal of Pharmaceutics, Volume 15, Issue 1, Pages $25-$ 35. [DOI]

Leuner, C. and Dressman, J. (2000). Improving drug solubility for oral delivery using solid dispersions. European Journal of Pharmaceutics and Biopharmaceutics, Volume 50, Issue 1, Pages 47-60. [DOI]
Makar, RR, Latif, R, Hosni, EA , Omaima, N. and Gazayerly, EI. (2013). Optimization for glimepiride dissolution enhancement utilizing different carriers and techniques. Journal of Pharmaceutical Investigation, Volume 43, Pages 115-131. [DOI]

Massimo, MB. (2003). Glimepiride in type 2 diabetes mellitus: a review of the worldwide therapeutic experience. Clinical Therapeutics, Volume 25, Issue 3, Pages 799-816.

Mehnert, W. and Mader, K. (2001). Solid lipid nanoparticles production, characterization, and applications. Advanced Drug Delivery Reviews, Volume 47, Issue 2-3, Pages 165-196. [DOI]

Mockel, JE. and Lippold, BC. (1993). Zero order release from hydrocolloid matrices. Pharmaceutical Research, Volume 10, Issue 7, Pages 10661070. [DOI]

Rajpurohit, VS., Rakha, P., Goyal, S., Dureja, H., Arorac, G. and Nagpal, M. (2011). Formulation and characterization of solid dispersions of glimepiride through factorial design. Iranian Journal of Pharmaceutical Sciences, Volume 7, Issue 1, Pages 7-16.

Reddy, MS., Fazalul, HSM. and Apte, SS. (2011). Solubility enhancement of fenofibrate, a BCS class II drug by self-emulsifying drug delivery systems. International Research Journal of Pharmacy, Volume 2, Issue 11, Pages 173-177.

Reddy, KR., Mutalik, S. and Reddy, S. (2003). Once-daily sustained matrix tablets of nicorandil formulation and in vitro evaluation. AAPS PharmTech Sciences, Volume 4, Issue 4, Pages E61. [DOI]

Reven, S., Grdadolnik, J., Kristl, J. and Zagar, E. (2010). Hyperbranched poly (esteramides) as solubility enhancers for poorly water soluble drug glimepiride. International Journal of Pharmaceutics, Volume 396, Issue 1-2, Pages 119-126. [DOI]

Sapkal, NP., Kilor, VA., Bhusari, KP. and Daud, AS. (2007). Evaluation of some methods for preparing gliclazide- $\beta$-cyclodextrin inclusion complexes. Tropical Journal of Pharmaceutical Research, Volume 6, Issue 4, Pages 833-840. [DOI]

Sarathchandiran, I. (2012). A review on nanotechnology in solid lipid nanoparticles. International Journal of Pharmaceutical Development and Technology, Volume 2, Issue 1, Pages 45-61.

Serajuddin, A. (1999). Solid dispersion of poorly water soluble drugs: early promises, subsequent problems and recent breakthroughs. Journal Pharmaceutical Sciences, Volume 88, Issue 10, Pages 1058-1066. [DOI]

Shohin, IE., Kulinich, JI., Ramenskaya, GV. and Vasilenko, GF. (2011). Evaluation of in vitro equivalence for drugs containing BCS class II compound ketoprofen. Dissolution Technologies, Volume 2, Pages 2629. [DOI]

Swathi, G., Prasanthi, NL., Manikiran, SS. and Ramarao, N. (2010). Solid lipid nanoparticles: colloidal carrier systems for drug delivery. International Journal of Pharmaceutical Science and Research, Volume 1, Issue 12, Pages 01-16.

Vidyadhara, S., Babu, JR., Sasidhar, RLC., Ramu, A., Prasad, SS. and Tejasree, M. (2011). Formulation and evaluation of glimepiride solid dispersions and their tablet formulations for enhanced bioavailability. Pharmanest, Volume 2, Issue 1, Pages 15-20.

Wagh, VT. and Wagh, RD. (2015). Solid dispersion techniques for enhancement of solubilization and bioavailability of poorly water soluble drugs - A Review. International Journal of Pharmacy and Technology. Volume 6, Issue 4, Pages 3027-3045.

Wei, H. and Lobenber, RL. (2006). Biorelevant dissolution media as a predictive tool for glyburide a class II drug. European Journal of Pharmaceutical Sciences, Volume 29, Issue 1, Pages 45-52. [DOI]

Yang, Y. (2000). Effect of preparation conditions on morphology and release profiles of biodegradable polymeric microspheres containing protein fabricated by double-emulsion method. Chemical Engineering and Science, Volume 55, Issue 12, Pages 2223-2236. [DOI]

Yogesh, ST., Indrajeet, DG. and Avinash, HH. (2011). Solubility enhancement techniques: a review on conventional and novel approaches. International Journal of Pharmaceutical Science and Research, Volume 2, Issue 10, Pages 2501-2513. 